\title{
Advantage of dual wavelength light-emitting diodes with dip-shaped quantum wells
}

\author{
XU YiQin, FAN GuangHan ${ }^{*}$, ZHOU DeTao, LI Xin, LU TaiPing, ZHAO Fang, \\ ZHANG YunYan, ZHENG ShuWen \& GONG ChangChun
}

Institute of Opto-Electronic Materials and Technology, South China Normal University, Guangzhou 510631, China

Received October 31, 2011; accepted January 5, 2012; published online May 22, 2012

\begin{abstract}
Dual-wavelength light-emitting diodes (DW-LEDs) with dip-shaped quantum wells have been studied by numerical simulation. The emission spectra, light output power, carrier concentration in the quantum wells and internal quantum efficiency are investigated. The simulation results indicate that the DW-LEDs with dip-shaped quantum wells perform better than conventional LEDs with rectangular quantum wells in terms of light output power, leakage current and efficiency droop. These improvements in the electrical and optical characteristics are mainly attributed to the alleviation of the electrostatic field in the dip-shaped quantum wells.
\end{abstract}

dip-shaped quantum wells, numerical simulation, dual-wavelength LED

Citation: $\quad \mathrm{Xu} \mathrm{Y} \mathrm{Q,} \mathrm{Fan} \mathrm{G} \mathrm{H,} \mathrm{Zhou} \mathrm{D} \mathrm{T,} \mathrm{et} \mathrm{al.} \mathrm{Advantage} \mathrm{of} \mathrm{dual} \mathrm{wavelength} \mathrm{light-emitting} \mathrm{diodes} \mathrm{with} \mathrm{dip-shaped} \mathrm{quantum} \mathrm{wells.} \mathrm{Chin} \mathrm{Sci} \mathrm{Bull,} \mathrm{2012,} \mathrm{57:}$ 2562-2566, doi: 10.1007/s11434-012-5211-2

Nitride-based light-emitting diodes (LEDs) have attracted considerable interest because of their lower power consumption, small size, longer lifetime and eco-friendly nature $[1,2]$. This solid light source may even replace the fluorescent lamp for general lighting in the future. The nitride-based dualwavelength light-emitting diodes (DW-LEDs) described here are composed of two kinds of quantum wells (QWs) that can emit light at different wavelengths. By adjusting the spectral intensity, the DW-LEDs could become phosphor-free whitelight LEDs, which would provide better performance in the solid-state white-light lighting area.

However, DW-LEDs have several serious restrictions affecting their applications, similar to the problems of conventional single wavelength LEDs. A well-known fundamental problem is referred to as efficiency droop [3-5]. The polarization field is widely acknowledged as the main reason for efficiency droop [6-8]. The conventional growth direction for GaN-based LEDs is along the polar (0001) axis, and this results in large piezoelectric and spontaneous

*Corresponding author (email: gfan@scnu.edu.cn) polarization because of the lack of inversion symmetry [9]. Differences in the indium components between the well layer and barrier layer cause lattice mismatch, which results in a large piezoelectric polarization in InGaN-based QWs. The piezoelectric effect eventually leads to a strong electrostatic field and a band bending situation in the active region, which leads to a reduction in both radiative recombination rate and internal quantum efficiency (IQE). DW-LEDs also have their own problems, which do not exist in single wavelength LEDs. Zhang et al. [10,11] reported that the shallow wells in DW-LEDs show poor carrier confinement performance, with the result that the radiative recombination rate in the shallow wells is much smaller than that in the deep wells, and thus the different intensities of the two peak wavelengths which are emitted by the two types of well become obvious in simulations using the conventional structure.

It was reported by Zhao et al. [12,13] that the single wavelength LEDs with dip-shaped QWs showed an improvement over conventional structures with rectangular QWs in the spontaneous recombination rate and output power. However, the DW-LED with dip-shaped QWs has 
not yet been reported, and the properties of the DW-LED with dip-shaped QWs have not previously been studied. In this work, dip-shaped quantum wells are used in the DWLED structure instead of the conventional rectangular QWs for the first time. The results reveal that the DW-LEDs with dip-shaped QWs can overcome the issues mentioned above.

\section{Numerical simulation}

The electrical and optical properties of two LED structures are investigated numerically with the APSYS simulation program, developed by Crosslight Software Inc (http://www. crosslight.com).

The conventional DW-LEDs were grown on a c-plane sapphire substrate, followed by a $3 \mu \mathrm{m}$-thick n-GaN layer (n-doping $=5 \times 10^{18} \mathrm{~cm}^{-3}$ ). The active region consists of four 2.5-nm-thick InGaN QWs, separated by five 10-nm-thick $\mathrm{GaN}$ barriers. The composition of the two QWs that are closer to the p-type hole-injection layer is $\operatorname{In}_{0.18} \mathrm{Ga}_{0.82} \mathrm{~N}$; while that of the other two QWs, which are closer to the n-type electron-injection layer, is $\operatorname{In}_{0.11} \mathrm{Ga}_{0.89} \mathrm{~N}$. On top of the active region, there are a 30 -nm-thick $\mathrm{p}-\mathrm{Al}_{0.1} \mathrm{Ga}_{0.9} \mathrm{~N}$ layer ( $\mathrm{p}$-doping $=5 \times 10^{17} \mathrm{~cm}^{-3}$ ) and a $0.15-\mu \mathrm{m}$-thick $\mathrm{p}$-GaN cap layer $\left(p\right.$-doping $=7 \times 10^{17} \mathrm{~cm}^{-3}$ ). The structure of the DWLED with dip-shaped QWs is the same as the conventional structure except for the active region, which consists of four dip-shaped QWs, separated by five 10-nm-thick GaN barri- ers. The composition of two QWs that are closer to the p-type hole-injection layer is $0.5 \mathrm{~nm}-\mathrm{In}_{0.05} \mathrm{Ga}_{0.95} \mathrm{~N} / 1.5 \mathrm{~nm}-\mathrm{In}_{0.18^{-}}$ $\mathrm{Ga}_{0.82} \mathrm{~N} / 0.5 \mathrm{~nm}-\mathrm{In}_{0.05} \mathrm{Ga}_{0.95} \mathrm{~N}$, while that of the other two QWs, which are closer to the n-type electron-injection layer, is $0.5 \mathrm{~nm}-\mathrm{In}_{0.05} \mathrm{Ga}_{0.95} \mathrm{~N} / 1.5 \mathrm{~nm}-\mathrm{In}_{0.11} \mathrm{Ga}_{0.89} \mathrm{~N} / 0.5 \mathrm{~nm}-\mathrm{In}_{0.05^{-}}$ $\mathrm{Ga}_{0.95} \mathrm{~N}$. The device structures are shown in Figure 1 .

The bandgap energies of InGaN and AlGaN ternary alloys can be expressed as [13]

$$
\begin{aligned}
E_{\mathrm{g}}\left(\mathrm{In}_{x} \mathrm{Ga}_{1-x} \mathrm{~N}\right)= & x \cdot E_{\mathrm{g}}(\mathrm{InN})+(1-x) \\
& \cdot E_{\mathrm{g}}(\mathrm{GaN})-1.4 x \cdot(1-x), \\
E_{\mathrm{g}}\left(\mathrm{Al}_{x} \mathrm{Ga}_{1-x} \mathrm{~N}\right)= & x \cdot E_{\mathrm{g}}(\mathrm{AlN})+(1-x) \\
& \cdot E_{\mathrm{g}}(\mathrm{GaN})-0.7 x \cdot(1-x),
\end{aligned}
$$

where $E_{\mathrm{g}}(\mathrm{InN}), E_{\mathrm{g}}(\mathrm{AlN})$ and $E_{\mathrm{g}}(\mathrm{GaN})$ are the bandgap energies of InN, AlN and GaN, with values of $0.78,6.25$ and $3.51 \mathrm{eV}$, respectively [14]. The internal absorption within the LED device is assumed to be $500 \mathrm{~m}^{-1}$ and the operating temperature is assumed to be $300 \mathrm{~K}$. To simplify the simulation, the light extraction efficiency is assumed to be 0.78 . Other material parameters of the semiconductors used in the simulation can be found in [15].

\section{Results and discussion}

Figure 2(a) shows the light output power as a function of
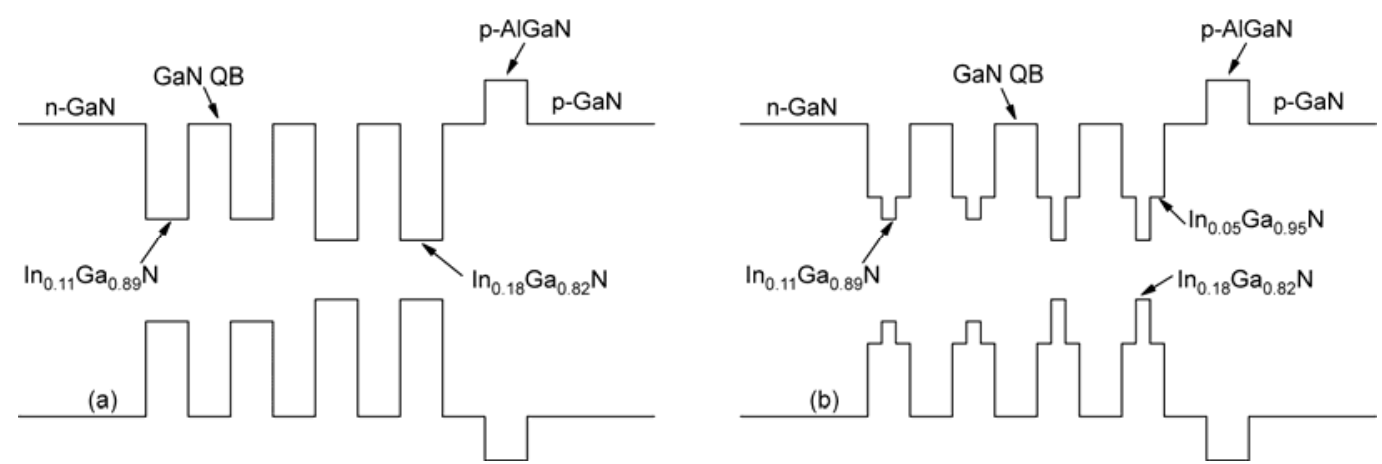

Figure 1 Schematic diagram of GaN-based LED structures, with rectangular QWs (a) and with dip-shaped QWs (b).
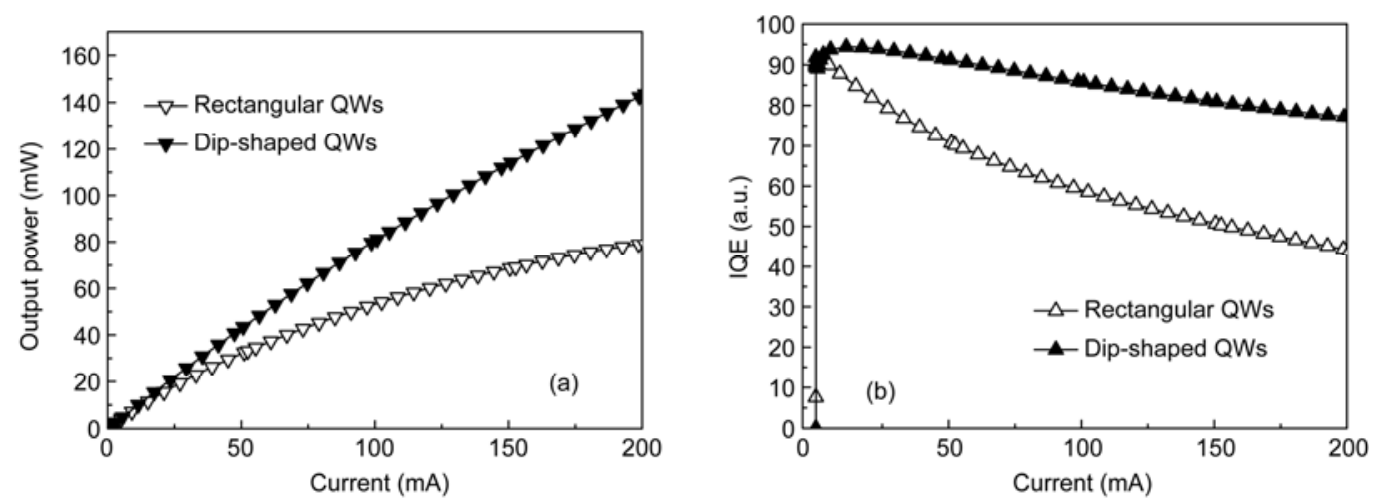

Figure 2 (a) Simulated light output power and (b) IQE as a function of current for the two LED structures. 
current for both LED structures, which indicates that the difference in light output power between the two structures is small at low injection currents, but the difference increases sharply with increasing injection current. The light output power of the conventional DW-LED with rectangular QWs increased slowly, while that of the newly designed DW-LED with dip-shaped QWs increased linearly with the increasing forward current. At $200 \mathrm{~mA}$, the output power reaches $143 \mathrm{~mW}$, which is almost twice that of the conventional structure. In Figure 2(b), an obvious efficiency droop could be seen in the conventional DW-LED as the forward current increased, while the IQE of the newly designed DW-LED with dip-shaped QWs decreased more slowly. Further analysis of the simulation indicates that the use of dip-shaped QWs in the DW-LED is beneficial in alleviating the electrostatic field, which efficiently improves the hole transport and increases the electron confinement; when the electrons can be confined efficiently, the leakage current can obviously be reduced. Therefore, the light output power of the newly designed DW-LED with dip-shaped QWs increased more rapidly than that of the conventional LED as the forward current increased. Figure 2 shows that by changing the rectangular QWs into dip-shaped QWs, the performance of the DW-LED improves remarkably, with increased output power and mitigation of the efficiency droop.

Figure 3(a) and (b) show the energy band diagram of the two structures at $200 \mathrm{~mA}$. In Figure 3(a), it is obvious that the conventional DW-LED structure with rectangular QWs has severely sloped triangular barriers, which will form obstacles to carrier transport into the active region. However, things are different in DW-LEDs with dip-shaped QWs. It is apparent from Figure 3(b) that the sloped triangular barriers are less severe because of the alleviation of the electrostatic fields in the active region. Because the dip-shaped QWs include three layers, i.e. a $1.5 \mathrm{~nm}-\mathrm{In}_{0.18} \mathrm{Ga}_{0.82} \mathrm{~N}$ or $1.5 \mathrm{~nm}$ $\mathrm{In}_{0.11} \mathrm{Ga}_{0.89} \mathrm{~N}$ layer sandwiched by two $0.5 \mathrm{~nm}$ thick $\mathrm{In}_{0.05^{-}}$ $\mathrm{Ga}_{0.95} \mathrm{~N}$ layers, the two inserted $\mathrm{In}_{0.05} \mathrm{Ga}_{0.95} \mathrm{~N}$ layers decrease the lattice mismatch between the well layers and the barrier layers, and thus the electrostatic field can be greatly reduced, which in turn results in a lower quantum confined Stark effect (QCSE) and better light emission efficiency. Also, a large electrostatic field causes charge separation, because the electrons and holes are pulled to opposite sides. Lu et al. [16] reported that the use of dip-shaped QWs could suppress the electrostatic field in the active region, and thus the overlap of the electron and hole wave functions can be markedly enhanced, from $33.1 \%$ to $47.4 \%$. With the increased overlap of the electron and hole wave functions, the radiative recombination rate and IQE will also improve.

We note from Figure 3(a) and (b) that the DW-LED with dip-shaped QWs has a lower potential barrier height in the valence band, which benefits the carrier transport in the active region. The quasi-Fermi levels of holes are mostly filled in the shallow wells and are much flatter, and it is anticipated that the hole concentration can be distributed more uniformly in the shallow QWs.

The carrier concentrations of the two structures in the active region at $200 \mathrm{~mA}$ are shown in Figure 4(a) and (b), which indicate that both the electron and hole distributions in the dip-shaped QWs are more uniform than those of the rectangular QWs. The carrier concentration is basically enhanced in the dip-shaped QWs, especially in the shallow $\left(\mathrm{In}_{0.11}\right)$ wells, while we can hardly see the hole concentration in the rectangular $\operatorname{In}_{0.11}$ well. Figure 4(c) shows the radiative recombination rates of the two structures. It is apparent that the radiative recombination of the conventional rectangular QWs mainly occurs in the deep $\left(\operatorname{In}_{0.18}\right)$ wells, and is almost zero in the shallow $\left(\operatorname{In}_{0.11}\right)$ wells, but it is much larger in the dip-shaped QWs because of the increased concentration of electrons and holes. As shown in Figure 4(d), it is too difficult to observe the short wave which is emitted by the shallow $\left(\mathrm{In}_{0.11}\right)$ wells in the conventional DW-LED because of their lack of carriers. As for the new design with dip-shaped QWs, the shorter wavelength of the spontaneous emission spectrum is much larger, which we attribute to the increase in the radiative recombination rate in the shallow $\left(\operatorname{In}_{0.11}\right)$ wells. We also see that the two peak wavelengths of the rectangular QWs and the dip-shaped QWs are $418 \mathrm{~nm} / 471 \mathrm{~nm}$ and $412 \mathrm{~nm} / 471 \mathrm{~nm}$ at $200 \mathrm{~mA}$, respectively.
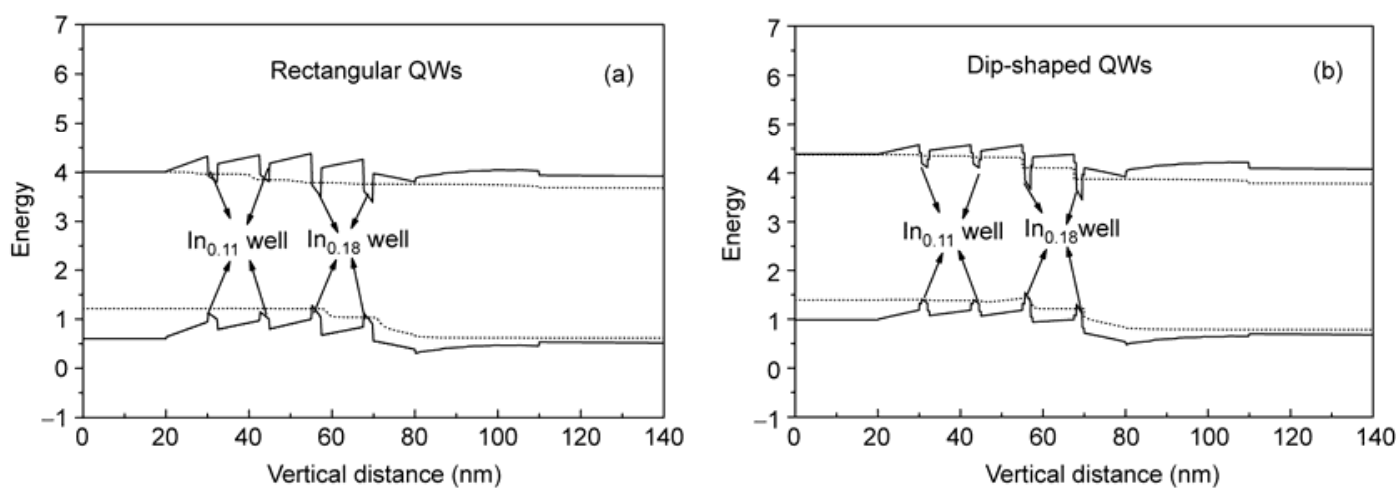

Figure 3 Energy band diagrams of LEDs with rectangular QWs (a) and dip-shaped QWs (b). 

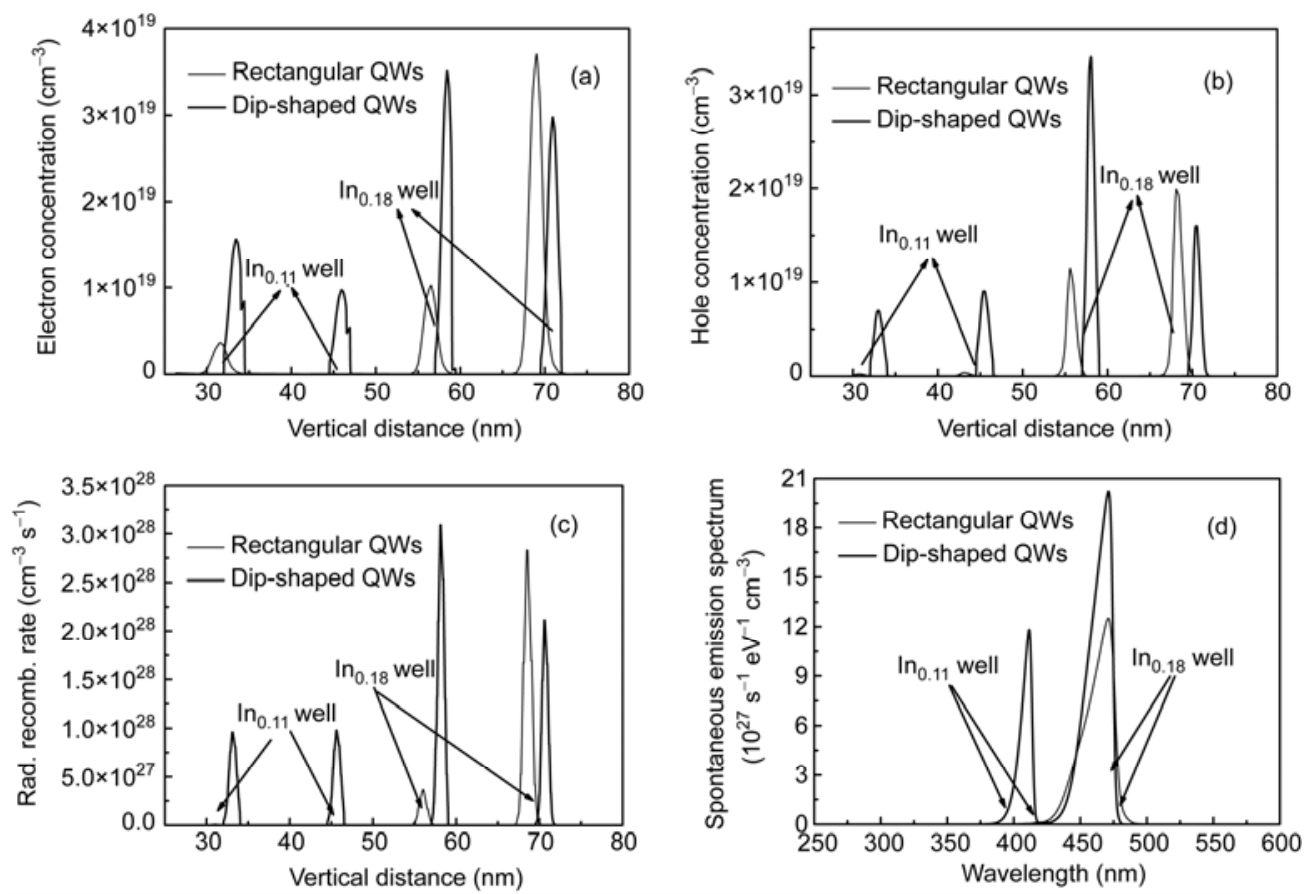

Figure 4 (a) Electron and (b) hole concentration distributions in QWs at forward current of $200 \mathrm{~mA}$; (c) the radiative recombination rates of the two structures at $200 \mathrm{~mA}$; (d) spontaneous emission spectrum of the two structures at $200 \mathrm{~mA}$ (there are small location excursions in the horizontal axes for better observation in (a)-(c)).

The recent research by Park's group showed that the dip-shaped QW structure had a smaller effective mass of heavy holes, a larger spontaneous emission coefficient and optical matrix elements produced by Kane's parameter [17], which may be one of the reasons for the improvement in the hole distribution and carrier confinement in the dip-shaped QWs. A DW-LED with dip-shaped QWs also has better matching of the lattice constants between the dip-shaped wells and barriers, and thus the electrostatic field can be greatly reduced and the sloped triangular barriers and wells can be effectively suppressed. The potential barrier height will therefore be lower, with the result that more holes can be transported into the active region, and the radiative recombination in the shallow $\left(\operatorname{In}_{0.11}\right)$ well strengthens.

As shown in Figure 5, the electron density in the first dip-shaped QW at a vertical distance of $30 \mathrm{~nm}$ is dramatically reduced, which can be attributed to much greater electron-hole recombination in these QWs compared to their rectangular counterparts. We also noticed that the dip-shaped QWs in the $\mathrm{n}-\mathrm{GaN}$ region have higher electron density than the rectangular QWs, which indicates that the holes recombine with the electrons in the MQWs more efficiently and fewer holes were injected into the $\mathrm{n}-\mathrm{GaN}$ area, thus inducing a higher electron current. Because of the large polarization field induced band bending in the active region, the QB and EBL can hardly block the electron overflow from the QWs to p-type layer, and thus the electron leakage current of the DW-LED with rectangular QWs is severe. However, it is different in DW-LEDs with dip-shaped QWs, where the

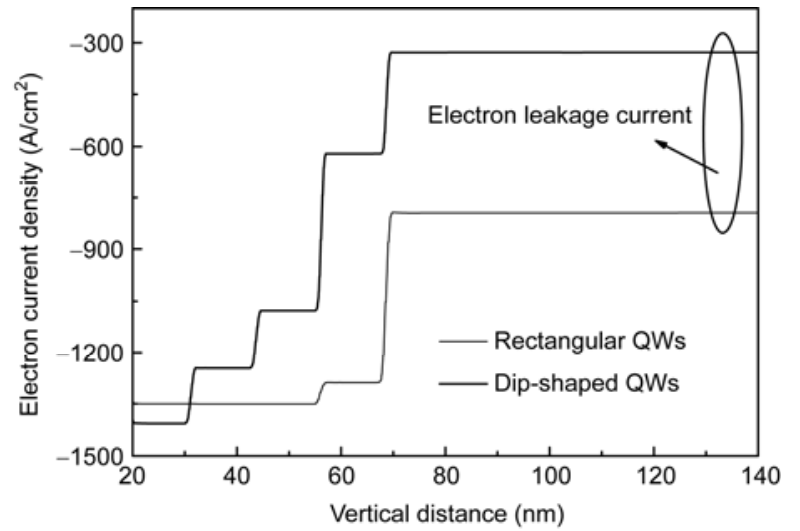

Figure 5 Simulated electron current density through the two LED structures under $200 \mathrm{~mA}$ forward current.

electrons can be confined efficiently and the electron leakage can be greatly reduced. The escaped electrons from the MQW active region can recombine with holes in the p-type region and thereby lower the LED efficiency. This shows that the electron leakage current caused by the polarization fields is one of the dominant mechanisms that result in efficiency droop.

\section{Conclusions}

In conclusion, the DW-LED with dip-shaped QWs can alleviate the electrostatic field of the device, which efficiently 
increases the electron confinement in the QWs, reduces the electron leakage current and raises the radiative recombination rate in the shallow wells. In contrast to the conventional DW-LED, the DW-LED with dip-shaped QWs reports higher light output power, lower current leakage and lower efficiency droop. Based on analysis through numerical simulations, these improvements in the electrical and optical characteristics of DW-LEDs with dip-shaped QWs are mainly attributed to the alleviation of the electrostatic field.

This work was supported by the Project for the Combination of Production and Research Guided by the Ministry of Education of Guangdong Province in 2009 (2009B090300338), the LED Industrial Projects of Special Funds for Strategic Emerging Industries in 2011, Guangdong Province (2010A081002005), and the National Natural Science Foundation of China (61176043).

1 Kern R S, Chen C H, Fletcher R M, et al. Hall-effect characterization of III-V nitride semiconductors for high efficiency light emitting diodes. Mater Sci Eng, 1999, B59: 211-217

2 Schubert E F, Kim J K. Solid-state light sources getting smart. Science, 2005, 308: 1274-1278

3 Li Y L, Huang Y R, Lai Y H. Efficiency droop behaviors of InGaN/ GaN multiple-quantum-well light-emitting diodes with varying quantum well thickness. Appl Phys Lett, 2007, 91: 181113

4 Kim M H, Schubert M F, Dai Q, et al. Origin of efficiency droop in GaN-based light-emitting diodes. Appl Phys Lett, 2007, 91: 183507

5 Schubert M F, Xu J, Kim J K, et al. Polarization-matched GaInN/ AlGaInN multi-quantum-well light-emitting diodes with reduced efficiency droop. Appl Phys Lett, 2008, 93: 041102
6 Kuo Y K, Tsai M C, Yen S H. Numerical simulation of blue InGaN light-emitting diodes with polarization-matched AlGaInN electronblocking layer and barrier layer. Opt Commun, 2009, 282: 4252-4255

7 Tao Y B, Chen Z Z, Zhang F F, et al. Polarization modification in InGaN/GaN multiple quantum wells by symmetrical thin low temperature-GaN layers. J Appl Phys, 2010, 107: 103529

8 Chang S P, Lu T C, Zhuo L F, et al. Low droop nonpolar GaN/InGaN light emitting diode grown on m-plane GaN substrate. J Electrochem Soc, 2010, 157: H501-H503

9 Takeuchi T, Sota S, Katsuragawa M, et al. Quantum-confined Stark effect due to piezoelectric fields in GaInN strained quantum wells. Jpn J Appl Phys, 1997, 36: L382-L382

10 Zhang Y Y, Fan G H. Theoretical study of GaN interval layers and quantum well barrier layers of different doping types in dual-wavelength LED (in Chinese). Acta Phys Sin, 2011, 60: 018502

11 Zhang Y Y, Fan G H, Zhang Y, et al. Effect of spectrum-controol in dual-wavelength light-emitting diode by doped GaN (in Chinese). Acta Phys Sin, 2011, 60: 028503

12 Zhao H P, Liu G Y, Li X H, et al. Growths of staggered InGaN quantum wells light-emitting diodes emitting at 520-525 nm employing graded growth-temperature profile. Appl Phys Lett, 2009, 95: 061104

13 Zhao H, Arif R A, Tansu N. Design analysis of staggered InGaN quantum wells light-emitting diodes at 500-540 nm. IEEE J Sel Top Quant Electr, 2009, 15: 1104-1114

14 Stringfellow G B, Craford M G. High Brightness Light Emitting Diodes. New York: Academic Press, 1997. 412

15 Vurgaftman I, Meyer J R. Band parameters for nitrogen-containing semiconductors. J Appl Phys, 2003, 94: 3675-3691

16 Lu T P, Li S T, Zhang K, et al. Simulation study of blue InGaN light-emitting diodes with dip-shaped quantum wells. Chin Phys, 2011, 10: 108504

17 Park S H, Ahn D, Koo B H, et al. Dip-shaped InGaN/GaN quantum-well light-emitting diodes with high efficiency. Appl Phys Lett, 2009, 95: 063507

Open Access This article is distributed under the terms of the Creative Commons Attribution License which permits any use, distribution, and reproduction in any medium, provided the original author(s) and source are credited. 